\title{
Evaluación de los atributos de atención primaria de salud en establecimientos del primer nivel atención de Ayacucho, Perú
}

\author{
Evaluation of primary care attributes at the first level of care in Ayacucho, Peru \\ Magaly Lizana 1,a, Estefany Ángela Flores ${ }^{1, a}$, Silvia Carhuayo Luján 1,b \\ RESUMEN \\ Objetivo: Evaluar los atributos de la Atención Primaria de Salud (APS) en cuatro establecimientos de primer nivel \\ de la ciudad de Ayacucho. Material y métodos: Estudio observacional, descriptivo, transversal, realizado entre el 5 y \\ 24 de febrero del 2018. Se utilizó como técnica la entrevista y como instrumento, la guía de la entrevista estructurada \\ Primary Care Assessment Tools versión brasileña corta en español para la evaluación de la Atención Primaria de \\ Salud Resultados: Los atributos con menores calificaciones de desempeño fueron: Enfoque Familiar, Orientación \\ Comunitaria y Primer Contacto-Accesibilidad; los que tuvieron mejores puntajes fueron: Coordinación, Primer \\ Contacto-Utilización y Globalidad. Existieron múltiples diferencias significativas entre centros de salud excepto \\ en Enfoque Familiar que percibida por todos los centros como deficiente Conclusiones: El cumplimiento de los \\ atributos de Coordinación-Sistema de Atención, Primer Contacto-Utilización y Globalidad-Servicios recibidos \\ fueron percibidos como los más eficientes; los atributos de Enfoque Familiar, Orientación Comunitaria y Primer \\ Contacto-Utilización fueron percibidos como deficientes.
}

PALABRAS CLAVE: Atención primaria de salud, atención integral de salud, calidad, acceso y evaluación de la atención de salud. (Fuente: DeCS BIREME).

\section{SUMMARY}

Objective: To evaluate primary care attributes at the four first level of care centers in Ayacucho, Peru. Methods: a cross-sectional study was carried-out from 5-24 of February, 2018. An interview using the short version of the structured Brazilian Primary Care Assessment Tool translated into Spanish was done. Results: The least qualified attributes were family focus, community orientation and first contact-accessibility, in contrast coordination and first contact-utilization and globality attained the highest qualification. Marked differences were found among the centers with the exception of family focus that was found deficient by all centers. Conclusions: Differences in the attributes were found among the first level centers evaluated.

KEYWORDS: Primary health care, comprehensive health care, quality, access and evaluation of health care. (Source: MeSH NLM).

\footnotetext{
1 Universidad Nacional de San Cristóbal de Huamanga. Ayacucho, Perú.

a Estudiante de 6to año de Medicina Humana

b Licenciada en Estadística
} 


\section{INTRODUCCIÓN}

La Atención Primaria de Salud (APS) es la provisión continua y de calidad de todo tipo de servicios de salud personal orientada a cubrir las necesidades básicas como diagnóstico, tratamiento, seguimiento y rehabilitación de pacientes $(1,2)$; en la actualidad se alcanza una demanda progresiva por estos servicios solicitando que sean más justos, equitativos, integradores y sobretodo de calidad $(3,4)$.

El Ministerio de Salud (MINSA) como órgano rector del sistema de salud ha venido implementando el Aseguramiento Universal en Salud como parte de la reforma del sector, para el 2015 un $78,9 \%$ de la población se encontraba afiliado al SIS (Seguro Integral de Salud) y podían acceder a un conjunto de prestaciones de Salud (5). El objetivo del MINSA se centra en las necesidades de la persona, familia y comunidad, dando énfasis a las acciones de promoción de la salud y prevención de la enfermedad sin descuidar las actividades de recuperación y rehabilitación (3). Dicho objetivo representa el 70 y $80 \%$ de las necesidades básicas más frecuentes en la atención de la salud de la población a lo largo de la vida. Por lo cual se ha implementado el "Plan Nacional de Fortalecimiento del Primer Nivel de Atención 20112021", que busca cumplir y fortalecer la APS (6).

Los desafíos a los que se enfrentan los servicios de salud por el incremento de enfermedades crónicas no transmisibles, envejecimiento de la población y un mayor número de asegurados SIS requieren que se enfatice su orientación hacia la calidad buscando una mejor capacidad de respuesta en todos los niveles de atención $(6,7)$.

De acuerdo a la Organización Mundial de Salud (OMS) el cumplimiento de ciertos atributos en la APS: Primer contacto, continuidad de la atención, coordinación de la atención, globalidad, enfoque familiar y orientación comunitaria, garantizan su calidad y serian la soluciona a la actual crisis de atención sanitaria $(8,9)$. Por ello es necesaria la visión científica al momento de evaluarlos, para así orientar políticas de salud más eficaces al momento de implementar programas como el actual Modelo de Atención Integral en Salud Basada en la Familia y Comunidad (MAIS-BFC) $(2,3)$.

En nuestro medio existen pocos estudios que evalúan la calidad de atención (10-12) y a nivel local no se reportaron estudios similares. Según un estudio sobre el impacto de la estrategia de atención integral de salud a poblaciones excluidas y dispersas, el estado de vida y salud de la población ayacuchana es deficitario, ello es reflejado en los altos índices de desnutrición crónica, mortalidad materna y los problemas de salud mental (13), denotando la necesidad de la evaluación de la APS en esta región.

El objetivo del estudio fue evaluar los atributos de la Atención Primaria de Salud en cuatro establecimientos de primer nivel de la ciudad de Ayacucho.

\section{MATERIAL Y MÉTODOS}

Estudio observacional, descriptivo y transversal, realizado en cuatro establecimientos de primer nivel del MINSA de la provincia de Huamanga, departamento de Ayacucho durante el mes de febrero del 2018, esta región cuenta con 10 establecimientos de primer nivel de atención, por lo que se seleccionó a azar cuatro de ellos: "Morro de Arica (I-2)", "Ñahuinpuquio (I-2)", "Miraflores (I-3)" y Carmen alto (I-3). Se realizó una entrevista estructurado según Primary Care Assessment Tools (PCAT) versión corta, brasileña, validada al español (14).

Muestra: Se realizó un muestreo por conveniencia. Se incluyeron 697 entrevistas, 172 del centro de salud "Morro de Arica (I-2)", 176 de "Ñahuinpuquio (I-2)", 173 de "Miraflores (I-3)" y 176 de Carmen alto (I-3)

Criterios de Inclusión: Ser usuario del centro de salud, haber hecho uso del establecimiento en el último año por lo menos dos veces, aceptar ser parte de la encuesta y edad mayor a 18 años.

Criterios de exclusión: Presentar algún síndrome neurológico o psiquiátrico, no comprender el idioma español o ser analfabeta

Variables: La variable primaria fue el Cumplimiento de atributos de la APS. Las secundarias fueron: Primer contacto (subdimensiones: Utilización y Acceso), Continuidad, Coordinación (subdimensiones: Sistema de atención y Sistema de información) Integralidad o Globalidad (subdimensiones: Servicios Disponibles y Servicios Recibidos), Enfoque Familiar y Orientación Comunitaria.

El indicador fue la frecuencia con la que el usuario valoró los atributos en el establecimiento. Las variables fueron respondidas en una escala con cinco opciones, de acuerdo a la existencia o no de un rasgo: 1 . No, en 
absoluto; 2. Es probable que no; 3. Es probable que sí; 4. Sí, sin duda; 5 . No se

Análisis estadístico Se utilizó estadística descriptiva para hallar las puntuaciones de los atributos y para la comparación de los resultados de los centros de atención primaria de salud, se usó el análisis de varianza y pruebas de comparaciones múltiples como la prueba de Scheffé; se consideró significativo un $\mathrm{p}<0,05$.

Consideraciones éticas: El protocolo fue revisado y aprobado por el comité de ética de Hospital Nacional Docente Madre Niño San Bartolomé. Antes de cada entrevista se informó al usuario del centro de salud los objetivos del estudio, solicitando por escrito su consentimiento informado.

\section{RESULTADOS}

La población estudiada estuvo conformada por 697 personas; $609(87,4 \%)$ fueron de sexo femenino, la distribución por edad muestra (tabla 1) que el 78,3\% tenía entre 21 y 50 años. El 45,6\% realizaba trabajos en el hogar; $20,7 \%$ trabajaban pero no recibían remuneración, 13,2\% eran estudiantes y sólo el 11,9\% tenían trabajo remunerado.

En la encuesta de evaluación de las funciones de la APS, los puntajes variaron de 2,5 a 10,0 en una escala de 10 puntos en todas las dimensiones (tabla 2). Usando como punto de corte siete, las mejores puntaciones fueron en las funciones de CoordinaciónSistemas de información, Primer Contacto-Utilización y Globalidad-Servicios recibidos. En las demás funciones, los puntajes promedios estuvieron por debajo de siete, indicando debilidades o situaciones que tienen margen de trabajo para conseguir mejoras. Los atributos con menores calificaciones de desempeño fueron Enfoque Familiar, Orientación Comunitaria y Primer Contacto-Acceso.

El análisis de varianza al comparar las diferentes medias de los atributos de APS encontró algunas diferencias significativas entre establecimientos de salud ( $p<0,05$ ), excepto en el Enfoque familiar, que en todos los centros de salud, no se encontró diferencia significativa (tabla 3 ).

- Primer Contacto-Utilización: el análisis de varianza muestra que existe diferencia significativa entre los puntajes promedios de los establecimientos de salud $(\mathrm{p}<0,05)$, a excepción de Miraflores con Ñahuanpuquio.

- Primer Contact-Acceso: el análisis de varianza muestra que hay diferencias significativa entre los establecimientos de Salud de Nahuinpuquio con Miraflores o con Morro de Arica $(\mathrm{p}<<0,05)$.

- Continuidad de Atención: Existe diferencia significativa en los puntajes promedios $(\mathrm{p}<<0,05)$, no así entre Carmen Alto con Miraflores, o con Morro de Arica.

- Coordinación- Sistema de Atención: Existe diferencia significativa entre Carmen Alto con Morro de Arica o con Nahuinpuquio y Miraflores con Ñahuipuquio, $(\mathrm{p}<<0,05)$.

- Coordinación-Sistemas de Información: Hay diferencia significativa entre los puntaje promedios exceptuando Miraflores con Carmen Alto, los cuales no muestran diferencias significativas $(\mathrm{p}=0.997)$.

- Globalidad-Servicios Disponibles: Existe diferencia entre los puntajes promedios

Tabla 1. Población encuestada de acuerdo a edad y sexo.

\begin{tabular}{|c|c|c|c|}
\hline \multirow{2}{*}{$\begin{array}{l}\text { Edad } \\
\text { (años) }\end{array}$} & \multicolumn{2}{|c|}{ Sexo } & \multirow{2}{*}{$\begin{array}{l}\text { Total } \\
\text { n (\%) }\end{array}$} \\
\hline & $\begin{array}{c}\text { Femenino } \\
\text { n (\%) }\end{array}$ & $\begin{array}{c}\text { Masculino } \\
\text { n (\%) }\end{array}$ & \\
\hline $18-20$ & $76(12,5 \%)$ & $13(14,8 \%)$ & $89(12,8 \%)$ \\
\hline $21-30$ & $227(37,3 \%)$ & $25(28,4 \%)$ & $252(36,2 \%)$ \\
\hline $31-40$ & $162(26,6 \%)$ & $19(21,6 \%)$ & $181(26,0 \%)$ \\
\hline $41-50$ & $95(15,6 \%)$ & $17(19,3 \%)$ & $112(16,1 \%)$ \\
\hline $51-60$ & $41(6,7 \%)$ & $9(10,2 \%)$ & $50(7,2 \%)$ \\
\hline $61-70$ & $8(1,3 \%)$ & $5(5,7 \%)$ & $13(1,9 \%)$ \\
\hline Total & $609(87,4 \%)$ & $88(12,6 \%)$ & $697(100,0 \%)$ \\
\hline
\end{tabular}


Tabla 2. Puntajes obtenidos en todas las dimensiones de las funciones de la APS.

\begin{tabular}{lccccc}
\hline FUNCIONES & Media & $\mathbf{D . E}$ & $\mathbf{P}_{\mathbf{2 5}}$ & $\mathbf{P}_{\mathbf{5 0}}$ & $\mathbf{P}_{\mathbf{7 5}}$ \\
\hline Primer Contacto-Utilización & 8,3 & 2,5 & 7,5 & 10,0 & 10,0 \\
Primer Contacto-Acceso & 5,6 & 1,9 & 4.3 & 6,2 & 6,2 \\
Continuidad de la atención & 6,1 & 1,3 & 5,0 & 6,2 & 6,9 \\
Coordinación-Sistema de Atención & 5,7 & 2,4 & 2,5 & 5,3 & 7,5 \\
Coordinación-Sistema de Información & 8,6 & 2,3 & 7,5 & 10,0 & 10,0 \\
Globalidad-Servicios Disponibles & 5,9 & 2,3 & 3,7 & 6,2 & 7,5 \\
Globalidad-Servicios Recibidos & 7,3 & 2,0 & 6,2 & 7,8 & 9,0 \\
Enfoque Familiar & 4,4 & 2,3 & 2,5 & 2,5 & 6,2 \\
Orientación comunitaria & 4,5 & 2,9 & 2,5 & 2,5 & 7,5 \\
\hline
\end{tabular}

Tabla 3. Evaluación de las funciones de la atención primaria de salud por centro de salud Ayacucho, 2018.

\begin{tabular}{lcccc}
\hline FUNCIONES & $\begin{array}{c}\text { Morro de } \\
\text { Arica }\end{array}$ & Miraflores & Ñahuinpuquio & $\begin{array}{c}\text { Carmen } \\
\text { Alto }\end{array}$ \\
\hline Primer contacto-Utilización & 6,8 & 9,4 & 9,4 & 7,5 \\
Primer Contacto-Acceso & 5,4 & 5,2 & 6,2 & 5,7 \\
Continuidad de la atención & 5,6 & 6,2 & 7,0 & 5,8 \\
Coordinación-Sistema de atención & 6,0 & 5,5 & 6,3 & 5,0 \\
Coordinación-Sistema de información & 7,4 & 9,4 & 8,4 & 9,5 \\
Globalidad-Servicios disponibles & 5,2 & 6,9 & 5,4 & 6,3 \\
Globalidad-Servicios recibidos & 6,1 & 7,6 & 7,3 & 8,3 \\
Enfoque familiar & 4,3 & 4,7 & 4,5 & 4,4 \\
Orientación comunitaria & 4,0 & 6,2 & 3,7 & 4,2 \\
\hline
\end{tabular}

correspondiente a servicios disponibles en los centros de salud de atención primaria, a excepción de Morro de Arica con Ñahuinpuquio, o Miraflores con Carmen Alto.

- Globalidad-Servicios Recibidos: Se muestra diferencia significativa entre los puntajes $(\mathrm{p}<<0,05)$, no así en Miraflores con Ñahuinpuquio, en los que no hay evidencia suficiente a un nivel de significación del $5 \%$ para afirmar que son diferentes.

- Enfoque Familiar: A un nivel de significancia del 5\%, no existe evidencia suficiente para afirmar que existe diferencia significativa entre los puntajes promedios referidos a la dimensión de Enfoque Familiar $(\mathrm{p}=0,370)$.

- Orientación Comunitaria: El análisis de varianza muestra que existe diferencia significativa, entre
Miraflores con Morro de Arica, o con Ñahuinpuquio, o con Carmen Alto $(\mathrm{p}<0,05)$.

\section{DISCUSIÓN}

Los datos evidencian que los atributos percibidos como deficientes son: Enfoque familiar, Orientación comunitaria, Primer Contacto-Accesibilidad, Globalidad-Servicios disponible, Continuidad de la atención y los atributos evaluados como eficientes son únicamente Coordinación-Sistema de Información, Primer Contacto-Utilización y Globalidad, existieron múltiples diferencias significativas entre centros de salud excepto Enfoque familiar que fue percibida por todos los centros como deficiente.

Las dimensiones de Enfoque familiar y Orientación comunitaria no tienen antecedente en la región ni en el Perú de haberse estudiado, y al ser calificada como deficiente por todos los centros deja en claro que este 
es un punto que se debe trabajar y reforzar, además de ser de vital importancia, accesible económicamente y de potenciales resultados para la actual crisis sanitaria $(7,16)$.

No se cuenta con otros estudios en la provincia de Huamanga acerca de los atributos de la APS. Dos estudios anteriores realizados en la ciudad de Lima perciben el atributo de Primer Contacto en grado medio $(10,15)$, cabe mencionar que no especifican en que subdimensión. Esto se diferencia con el presente estudio porque Primer ContactoUtilización es percibida con un puntaje alto y Primer Contacto-Acceso con un puntaje bajo, la diferencia de resultados pueden ser debido a la fusión de los datos de las subdimensiones para el análisis estadístico de los estudios anteriores sesgando así los resultados, los resultados implican que los usuarios acuden primero a un establecimiento de salud ante un problema nuevo; sin embargo estos sistemas no son de fácil acceso para la población $(7,17)$.

Coordinación fue percibida por más de $60 \%$ de la población como de grado medio en un estudio anterior (15), cabe resaltar que no especifica en que subdimensión; en el presente trabajo CoordinaciónSistemas de información fue calificada de alto puntaje, esto se traduce en que la disponibilidad de instrumentos de registro de la información (historias clínicas) y de incorporación de esa información al plan de atención de la persona es percibida como eficiente (7).

Globalidad fue percibida como baja o deficiente, en otros estudios $(10,15)$, mientras que en el presente trabajo se obtuvieron puntajes altos, esto puede explicarse a las distintas poblaciones a las que se les hizo el estudio también al tiempo en que fueron hechos los estudios, ya que desde el 2011 se está implementando el Plan de Fortalecimiento del Primer Nivel de Atención progresivamente.

Los usuarios ante un problema nuevo de salud, primero acuden a un centro de APS antes que a otras alternativas, sin embargo, estos centros no son accesibles; la comunicación entre los centros de APS y su población es deficiente pues no se consulta sobre nuevas políticas que se puedan aplicar ni se pide opinión acerca de ello, no se contextualiza al paciente dentro de su familia durante la atención, el sistema de información de cada APS es eficaz (historias clínicas cómodamente disponibles); los servicios recibidos por cada usuario en consulta externa abordan temas que no solo se refieren a el problema por el cual se acudió al centro de APS, sino que abordan otros temas como hábitos saludable, control de demás medicamentos recibidos, entre otros.

En el presente estudio solo tres de los nueve atributos de la APS se percibieron eficientes, estos fueron Coordinación-Sistema de Atención, Primer Contacto-Utilización y Globalidad-Servicios recibidos, los atributos considerados como los más deficientes fueron Enfoque Familiar, Orientación Comunitaria y Primer Contacto-Accesibilidad.

Cabe mencionar que este estudio solo muestra la perspectiva del usuario y los resultados de una pequeña población; por lo que no se puede extrapolar, no hay límites establecidos entre la evaluación del programa o la evaluación de su aplicación.

\section{Agradecimientos:}

Al Doctor Fernando Ramón Vázquez, Doctor Christian Mejía Álvarez, Jesús Miguel Paucar Prado, Elmer Pérez Pozo y a los Centros de Salud que nos abrieron las puertas para poder realizar este trabajo.

\section{Declaración de financiamiento y de conflictos de interés:}

El estudio fue financiado por los autores; declaran no tener conflictos de interés.

\section{Contribución de autoría:}

ML: Participó en la concepción y diseño del trabajo, recolección/obtención de resultados, análisis e interpretación de datos, redacción del manuscrito, aporte de pacientes o material de estudio. EAF: Participó en la recolección/obtención de resultados, redacción del manuscrito, revisión crítica del manuscrito, aporte de pacientes o material y aprobación de la versión final. SCL: Participó en la recolección/obtención de resultados, revisión crítica del manuscrito, asesoría en estadística y aprobación de la versión final.

\section{Correspondencia:}

\section{Magaly Lizana Medrano}

Av. Salvador Cavero 371 "Las Nazarenas".

Ayacucho, Perú.

Correo electrónico: Magaly.lizana@hotmail.com

Celular: 953286653 


\section{REFERENCIAS BILIOGRÁFICAS}

1. Organización Mundial de la Salud. Conferencia internacional de Atención primaria de la Salud Declaración de Alma-Ata, 1978. Ginebra: Organización Mundial de la Salud; 2010. (Citado el 5 de diciembre del 2016) Disponible en: http:// www2.paho.org/hq/dmdocuments/2010/APS_Alma_ Ata-Declaracion-1978.pdf?ua=1

2. Instituto Suramericano de Gobierno en Salud. Mapeo y Análisis de los Modelos de Atención Primaria a la Salud en los Países de América del Sur: Mapeo de la APS en Perú. Rio de Janeiro: Instituto Suramericano de Gobierno en Salud; 2015. (Citado el 5 de diciembre del 2016) Disponible en: http://www.isags-unasur. org/uploads/eventos/ev[347]ling[1]anx[324].pdf

3. Ministerio de Salud. Modelo de Atención Integral de Salud Basado en la Familia y Comunidad. Lima: Ministerio de Salud; 2011. (Citado el 13 de enero del 2017) Disponible en: http://bvs.minsa.gob.pe/ local/MINSA/1617.pdf

4. Organización Mundial de salud. La Atención Primaria de Salud más necesaria que nunca. Ginebra: Organización Mundial de salud; 2008. (Citado el 2 de marzo del 2016) Disponible en: http://www.who. int $/$ whr/2008/08_overview_es.pdf?ua $=1$

5. Ministerio de Salud. Políticas y acciones en salud hacia el bicentenario. Documento de Gestión 2015. Lima: Ministerio de Salud; 2015. (Citado el 13 de enero del 2017) Disponible en: http://bvs.minsa.gob. pe/local/MINSA/3476.pdf

6. Ministerio de Salud. Plan Nacional de Fortalecimiento del Primer Nivel de Atención 2011-2021. Dirección General de Salud de las Personas. Lima: Ministerio de Salud; 2011. (Citado el 13 de enero del 2017) Disponible en: http://bvs.minsa.gob.pe/local/minsa/ 1620.pdf

7. Berra S. El estudio de las funciones de la atención primaria de salud. Córdoba: Facultad de Ciencias Médicas; Universidad Nacional de Córdoba; 2012. (Citado el 15 de Marzo del 2017) Disponible en: http://ciess.webs.fcm.unc.edu.ar/files/2012/05/2012 FuncionesAPS_AR-PCAT_CIESS-UNC.pdf

8. Ponzo J, Berra S, Giraldo-Osorio A, et al. Armonización iberoamericana de los instrumentos PCAT para la evaluación del primer nivel de atención. Rev Panam Salud Pública. 2018;42: e80. Doi: https:// doi.org/10.26633/RPSP.2018.80

9. Ministerio de Salud. Estándares de Calidad para el Primer Nivel de Atención en Salud. Lima, Perú: Ministerio de Salud; 2002. (Citado el 15 de Marzo del 2017) Disponible en: http://www.diresacusco. gob.pe/administracion/serums/Plan\%20Mejora\%20 Web/Estandar1erNivel2003.pdf
10. Cuba-Fuentes M, Jurado G, Estrella E. Evaluación del cumplimiento de los atributos de la Atención Primaria y grado de satisfacción de los usuarios de un establecimiento de primer nivel de atención. Rev Med Hered. 2011; 22(1):4-9. DOI: https://doi. org/10.20453/rmh.v22i1.1093

11. SolariL.Atención primaria de la salud:Uncompromiso pendiente. Revperúmedexpsaludpública.2013;30(2): 173-174. (Citado el 15 de Marzo del 2020) Disponible en: http://www.scielo.org.pe/scielo.php?script=sci arttext\&pid=S1726-46342013000200002\&lng=es .

12. Polo O. Experiencia en la implementación del Modelo de Atención Integral de Salud basado en Familia y Comunidad desde la Estrategia Sanitaria Nacional de Salud Familiar. Rev peru ginecol obstet. 2018; 64(3):375-382. Doi: 10.31403/rpgo.v64i2100

13. Bautista C. Estudio de impacto de la estrategia de atención integral de salud a poblaciones excluidas y dispersas - AISPED en la Región Ayacucho. Tesis de Grado: Lima, Perú: Universidad Nacional Mayor de San Marcos; 2011. 161pp. (Citado el 15 de Marzo del 2020) Disponible en: http://cybertesis. unmsm.edu.pe/bitstream/handle/cybertesis/857/ Bautista_pc.pdf? sequence $=1 \&$ isAllowed $=\mathrm{y}$

14. Vázquez F. Comportamiento psicométrico de una versión corta del PCAT en español. Aten Primaria. 2016; 49(2): 69-76. (Citado el 15 de Marzo del 2020) Disponible en: https://www.sciencedirect.com/ science/article/pii/S0212656716301792?via\%3Dihub

15. Quispe R. Cumplimiento de los Atributos de la Atención Primaria y Percepción de la calidad de los usuarios en Consulta Externa del Centro Materno Infantil José Gálvez VMT - Lima, 2018. Tesis de Grado. Trujillo: Universidad Cesar Vallejo; 2018. 123 pp. (Citado el 15 de Marzo del 2020) Disponible en: http://repositorio.ucv.edu.pe/bitstream/handle/ UCV/30378/Quispe_MR.pdf? sequence $=1 \&$ is Allowed $=\mathrm{y}$

16. García-Huidobro D. Enfoque familiar en la Atención Primaria: Una propuesta para mejorar la salud de todos. Rev Med Chile. 2010; 138: 1463-1464. (Citado el 15 de Marzo del 2020) Disponible en: https://scielo.conicyt.cl/scielo.php?script=sci_arttext \&pid=S0034-98872010001200019

17. Rosas A, Narciso V, Cuba M. Atributos de la Atención Primaria de Salud (A.P.S): Una visión desde la Medicina Familiar. Acta méd. Peruana. 2013; 30(1): 42-47. (Citado el 15 de Marzo del 2020) Disponible en: http://www.scielo.org.pe/scielo.php?script=sci_ar ttext\&pid=S1728-59172013000100008

Recibido: 30/03/2019

Aceptado: 28/05/2020 